\title{
Muscle torque production and kinematic properties in post-stroke patients: a pilot cross-sectional study
}

\author{
MATEUSZ KowaL ${ }^{1}$, ANNA KOlCZ ${ }^{1,2}$, ROBERT DYMAREK ${ }^{3 *}$, \\ MALGORZATA PAPROCKA-BOROWICZ ${ }^{1,2}$, JAN GNUS ${ }^{1}$

\footnotetext{
${ }^{1}$ Department of Physiotherapy, Faculty of Health Sciences, Wroclaw Medical University, Wrocław, Poland. ${ }^{2}$ Department of Neurological Rehabilitation, Provincial Specialist Hospital, Research and Development Centre, Wrocław, Poland. ${ }^{3}$ Department of Nervous System Diseases, Faculty of Health Sciences, Wroclaw Medical University, Wrocław, Poland.
}

\begin{abstract}
Purpose: Stroke-related hemiplegia is an important factor influencing parameters of gait. So far, limited papers have assessed temporo-spatial capabilities and their correlations with gait parameters in the early post-stroke stage. This pilot study evaluated the temporospatial parameters of gait and assessed the maximal isometric and isokinetic torque production of the plantar flexor and dorsiflexor muscles. Methods: 15 patients with lower limb spasticity and 15 healthy controls were included. Stroke severity was assessed using the Modified Ashworth Scale and the Barthel Index. Gait cadence, gait speed, and gait cycle were assessed using inertial sensors during a Timed Up and Go test. Maximal isometric and isokinetic torque production of the ankle plantar flexor and dorsiflexor muscles were assessed using an isokinetic dynamometer device. Results: Post-stroke patients had statistically significantly lower gait cadence than healthy participants $(17 \%, p<0.05)$. Statistically significantly lower values of vertical acceleration were also noted during a sit-to-stand movement task $(42 \%, p<0.05)$. Plantar flexion torque of the affected limb was significantly different during isometric $(63 \%, p \leq 0.01)$ and isokinetic work for $30 \% \mathrm{~s}(49 \%, p=0.04), 60 \% \mathrm{~s}(58 \%, p=0.01)$ and $20 \% \mathrm{~s}(53 \%, p=0.01)$. Dorsiflexor muscles' torque production was significantly different in isometric activity $(38 \%, p=0.04)$. A statistically significant positive correlation occurred between the absolute peak torque of the dorsiflexor muscles in both static and speed phases of gait $(R s=0.65, p=0.04)$. Conclusions: Despite the low intensity of spasticity and early phase after stroke, differences in the muscle torque production and temporo-spatial parameters, as well as the correlations between them, were noticeable.
\end{abstract}

Key words: biomechanics, gait parameters, isokinetic assessment, neurorehabilitation, post-stroke hemiplegia, torque production

\section{Introduction}

Hemiplegia is a common, long-term impairment reducing gait performance after a stroke [1]. Hemiplegia leads to a lack of independent motor control of the muscle groups and incorrect co-activation of muscles acting on the joints of the lower limbs [2]. These disorders can be caused by changes in the number and method of recruitment of motor units. Such adverse conditions result in impaired motor control during the gait cycle [3].

The ankle plantar flexor muscles generate the forces needed to initiate gait [8] while the ankle dorsi- flexor muscles allow for alternate walking [4]. Asymmetrical torque production for muscles acting on the ankle joints may cause gait asymmetry. Kinematic properties of gait in persons after a stroke means change in the ranges of motion and this is reflected in gait phases, gait cadence, and gait speed, as well as limb load times during the stance and swing phases (temporo-spatial parameters) [5].

Kim et al. [6] reported that muscle torque may account for 66 to $72 \%$ of the variation in the temporospatial parameters of gait after a stroke. In post-stroke recovery, apart from asymmetrical gait cycles, people also exhibit asymmetrical muscle torque acting on the lower limb joints, which causes difficulties when

\footnotetext{
* Corresponding author: Robert Dymarek, Department of Nervous System Diseases, Faculty of Health Sciences, Wroclaw Medical University, ul. Bartla 5, 51-618 Wrocław, Poland. Phone: +48 7178418 39, Fax:+48 7134593 24, e-mail: r.dymarek@gmail.com Received: October 7th, 2019

Accepted for publication: December 9th, 2019
} 
changing position from sitting to standing [7]. This activity requires increased muscle torque production and a greater range of motion in the joints than walking [8]. In people at an early stage after a stroke, the first attempts to change positions from sitting to standing are a key barrier to be overcome in order to reeducate gait in the future. Lomaglio et al. [9] showed statistically significant correlations in post-stroke patients between the torque of the plantar flexor muscles on the affected side and the movement task in a Timed Up and Go test (TUG) $(r=-0.466)$.

Moon et al. [10] concluded that greater emphasis should be placed on generating ankle muscle torque to improve gait functions, while Freire et al. [11] reported that morphological changes in the Achilles tendon in hemiparetic stroke survivors_were not statistically significant, they also noted differences in the plantar flexor muscles' torque between the affected and the healthy limb. Rabelo et al. [12] showed that the correlation between the torque of muscles acting on ankle joints and the gait parameters of people after a stroke can be useful for monitoring differences in the torque of muscles acting on the ankle joint and can be helpful in programming rehabilitation to improve muscle strength in chronic, post-stroke hemiparesis.

In the daily clinical practice of the therapeutic team, there are a limited number of measurement tools that allow for objectively monitoring the improvement progress of individuals after a stroke. In the early stages of rehabilitation of people with low motor deficits, future compensations are difficult to predict, especially as their consequences last for many years. This study evaluated gait disorders following an early post-stroke period based on differences in the temporo-spatial parameters of gait components, TUG test and the torque of the ankle joint [13].

Previous studies have examined this significant research and clinical problem [14]. These differences in muscle torque production in early post-stroke period can serve as criteria to differentiate correct movement patterns from pathological ones, as well as tools to evaluate the rehabilitation process. After an early stage, a stroke becomes chronic. Therefore, there are a large number of potential outcomes of a stroke that require detailed examination. It has been hypothesized that incensement in the strength of muscles acting on the ankle joint may improve gait patterns. This improvement can be obtained by introducing resistance exercises to the rehabilitation process of post-stroke patients. Although the dynamometric device used to assess muscle strength is not a standard in clinical practice, the results of the study should encourage practitioners to use objective methods of assessing muscle strength even in people with small neurological deficits.

It should be pointed out that, to the best of our knowledge, the current body of literature lacks studies determining the correlations between ankle joint muscles in static and dynamic conditions at different angular velocities for plantar flexors and dorsiflexors, as well as gait speed in people during an early poststroke period after a stroke. So far, only a correlation between gait asymmetry and muscle torque in patients with chronic stroke has been shown [6]. In our study, we expected to observe the same relation among patients being in an early post-stroke period.

Therefore, this research has aimed to find out the power-velocity capabilities of muscles acting on the ankle joint at low, medium, and high-speed angular velocities for ankle plantar flexor and dorsiflexor muscles, on both the affected and unaffected sides, in people in early post-stroke period. We also aimed at evaluation of the motor task of TUG, temporo-spatial variables of gait and correlation between the torque of muscles acting on ankle joints and gait speed.

\section{Materials and methods}

\subsection{Study design and ethics}

This study was carried out as a cross-sectional study. The Strengthening the Reporting of Observational Studies in Epidemiology (STROBE) guidelines for observational studies have been followed [15]. The research project was approved by the Bioethics Committee of Wroclaw Medical University, Poland (approval no. KB-232/2016). All patients in the study were informed of its purpose and course and of their right to withdraw at any stage. All patients provided signed, informed consent at the beginning of the study.

\subsection{Study participants}

The study was conducted from March 2018 to November 2018 at the Department of Neurological Rehabilitation of the Provincial Specialist Hospital in Wrocław, Poland. In the present study, 30 participants were enrolled (14 male and 16 female). The post-stroke group consisted of 15 patients $(7$ male and 8 female, mean age of $57 \pm 11$ years old), and the time since stroke was $1.6 \pm 0.6$ months. The study group had 
a low level of disability. The Modified Ashworth Scale (MAS) was used to assess one of the symptoms of damage to the central nervous system, which is spastic muscular tension. The level of functional disability is assessed according to Barthel Index (BI). The control group consisted of 15 healthy people ( 7 male and 8 female, mean age of $32 \pm 4$ years old). For the participants in the control group, gait analysis and a torque measurement of muscles acting on ankle joints were collected, and available literature data indicating the reference values for healthy people were used respectively [16]. The detailed demographic and clinical characteristics of the study participants are shown in Tables 1 and 2.

Table 1. Demographic characteristics of study participants $(\mathrm{M} \pm \mathrm{SD})$

\begin{tabular}{|l|c|c|}
\hline \multicolumn{1}{|c|}{ Characteristics } & Post-stroke $(N=15)$ & Healthy $(M=15)$ \\
\hline Age [years] & $57.2 \pm 11$ & $32.3 \pm 4$ \\
\hline Male/female & $7 / 8$ & $7 / 8$ \\
\hline Body mass $[\mathrm{kg}]$ & $81.3 \pm 21$ & $80.1 \pm 9$ \\
\hline Height $[\mathrm{cm}]$ & $164.3 \pm 11$ & $167.4 \pm 10$ \\
\hline
\end{tabular}

Abbreviations: $M$ - mean, SD - standard deviation, $N$ - number of participants.

\subsection{Qualification procedures}

Patients were selected for the study via medical documentation and a medical examination performed by an experienced neurologist. The inclusion criteria were: the normal range of motion values for both ankle joints, no decreased strength of the muscles acting on ankle joints in a physical examination, and no cognitive or mental disorders. The exclusion criteria were: a level of spasticity higher than grade one on the MAS, severe limb pain, sensory impairment, visual impairment, cognitive impairment, balance disturbances, the presence of other neuromuscular or musculoskeletal disorders, and the inability to independently walk a distance of at least ten meters.

\subsection{Gait analysis}

To assess the temporo-spatial variables, an inertial sensor, the BTS G-Sensor (BTS Bioengineering Corp., Quincy, MA, USA), was used. The sensor was equipped with a triaxial 16-bit accelerometer with multiple sensitivity ( $\pm 2, \pm 4, \pm 8, \pm 16 \mathrm{~g})$, a triaxial 16-bit gyroscope

Table 2. Clinical characteristics in post-stroke patients

\begin{tabular}{|c|c|c|c|c|c|c|c|}
\hline No. & $\begin{array}{l}\text { Time since } \\
\text { stroke } \\
{[\text { month }]}\end{array}$ & Sex & $\begin{array}{l}\text { Stroke } \\
\text { etiology }\end{array}$ & $\begin{array}{l}\text { Affected } \\
\operatorname{limb}\end{array}$ & MAS & $\begin{array}{c}\text { Limb } \\
\text { domination }\end{array}$ & BI \\
\hline 1 & 1 & $M$ & IS & $\mathrm{R}$ & 1 & $\mathrm{R}$ & 80 \\
\hline 2 & 2 & $\mathrm{~F}$ & IS & $\mathrm{L}$ & 1 & $\mathrm{R}$ & 80 \\
\hline 3 & 3 & $\mathrm{M}$ & IS & $\mathrm{L}$ & 1 & $\mathrm{R}$ & 85 \\
\hline 4 & 2 & $\mathrm{~F}$ & IS & $\mathrm{L}$ & 1 & $\mathrm{R}$ & 80 \\
\hline 5 & 1 & $\mathrm{M}$ & IS & $\mathrm{L}$ & 0 & $\mathrm{R}$ & 80 \\
\hline 6 & 2 & $\mathrm{M}$ & IS & $\mathrm{R}$ & 1 & $\mathrm{R}$ & 80 \\
\hline 7 & 1 & $\mathrm{~F}$ & IS & $\mathrm{R}$ & 1 & $\mathrm{R}$ & 80 \\
\hline 8 & 2 & $\mathrm{~F}$ & HS & $\mathrm{R}$ & 1 & $\mathrm{R}$ & 80 \\
\hline 9 & 1 & $\mathrm{M}$ & IS & $\mathrm{L}$ & 0 & $\mathrm{R}$ & 80 \\
\hline 10 & 2 & $\mathrm{M}$ & IS & $\mathrm{R}$ & 1 & $\mathrm{R}$ & 85 \\
\hline 11 & 1 & $\mathrm{M}$ & IS & $\mathrm{R}$ & 1 & $\mathrm{R}$ & 80 \\
\hline 12 & 1 & $\mathrm{~F}$ & IS & $\mathrm{L}$ & 1 & $\mathrm{R}$ & 80 \\
\hline 13 & 1 & $\mathrm{M}$ & IS & $\mathrm{R}$ & 0 & $\mathrm{R}$ & 80 \\
\hline 14 & 2 & $\mathrm{~F}$ & HS & $\mathrm{R}$ & 1 & $\mathrm{R}$ & 85 \\
\hline 15 & 1 & $F$ & IS & $\mathrm{L}$ & 1 & $\mathrm{R}$ & 80 \\
\hline $\mathrm{M} / \mathrm{N}$ & 1.53 & & & & 0.80 & & 81.00 \\
\hline $\mathrm{SD}$ & 0.64 & & & & 0.41 & & 2.07 \\
\hline $\mathrm{Me}$ & 1.00 & $\mathrm{~F}=7 / \mathrm{M}=8$ & $\mathrm{IS}=13 / \mathrm{HS}=2$ & $\mathrm{R}=8 / \mathrm{L}=7$ & 1.00 & $\mathrm{R}=15 / \mathrm{L}=0$ & 80.00 \\
\hline Min & 1.00 & & & & 0.00 & & 80.00 \\
\hline Max & 3.00 & & & & 1.00 & & 85.00 \\
\hline
\end{tabular}

Abbreviations: IS - ischemic stroke, HS - hemorrhagic stroke, MAS - Modified Ashworth Scale, BI - Barthel Index, $\mathrm{R}$ - right, L - left, $N$ - number of participants, SD - standard deviation, Me - median, Min - minimum; Max - maximum. 
with multiple sensitivity $( \pm 250, \pm 500, \pm 1000, \pm 2000 \%$ s), and a triaxial 13-bit magnetometer $( \pm 1200 \mu \mathrm{T})$. An accelerometer recorded acceleration in three axes (vertical, antero/posterior and medio/lateral). The G-Sensor has been found valid for assessing physical activity in healthy adults, with an inter-instrument correlation coefficient between 0.90 and 0.99 and an intrainstrument coefficient of variation of $\leq 2.5 \%$ [17]. The output data were converted into numeric units on one axis or as a composite vector (i.e., the result of the sizes of all three axes). This study used the data of composite vectors recording selected temporo-spatial variables characterizing gait.

Measurements were always performed by the same researcher. The research procedure began by measuring the weight and height of patients, as well as the absolute length of their lower limbs (i.e., the greater trochanter of the femur to the lateral malleolus of the lower leg). The data were collected during two movement tasks - namely, gait with self-selected speed and the TUG test. An inertial sensor was placed on the patient's body according to the manufacturer's instructions (i.e., at the level of the sacral bone $\left(S_{1}\right)$ for gait analysis and the level of the lumbar spine $\left(\mathrm{L}_{2}\right)$ for TUG). Measurements were undertaken in footwear without the use of any additional orthopedic equipment. Gait assessments were performed during a movement task in which the subject walked a distance of seven meters. This distance ensured free and full communication between the sensor and the control unit and enabled us to register about two to four full gait cycles, depending on the patient's gait speed. Repetitions of this movement task were recorded for further analysis of the mean course and to extract the pattern of representative data. The selected temporo-spatial parameters analyzed were: gait cadence (GCAD) [steps/min], gait speed (GSP) $[\mathrm{m} / \mathrm{s}]$, and gait cycle (GC) for the single stance phase [\%]. A single stance/ support time ratio measured as a ratio of a single stance time to gait cycle time and expressed in percent.

The TUG test was measured by vertical acceleration (VTA) $\left[\mathrm{m} / \mathrm{s}^{2}\right]$ during sit-to-stand and standto-sit activities. The stabilization period, identified by Schenkman et al. [18], was not included in the time to complete the TUG. Individuals were seated on an armless chair with the lower limbs flexed at the hip and knee joints at approximately $90^{\circ}$. The distance from the feet to the chair was not defined. The subject's task was to stand up from the chair (without any upper limb assistance), walk a distance of about three meters, and then return to the seat (Fig. 1).

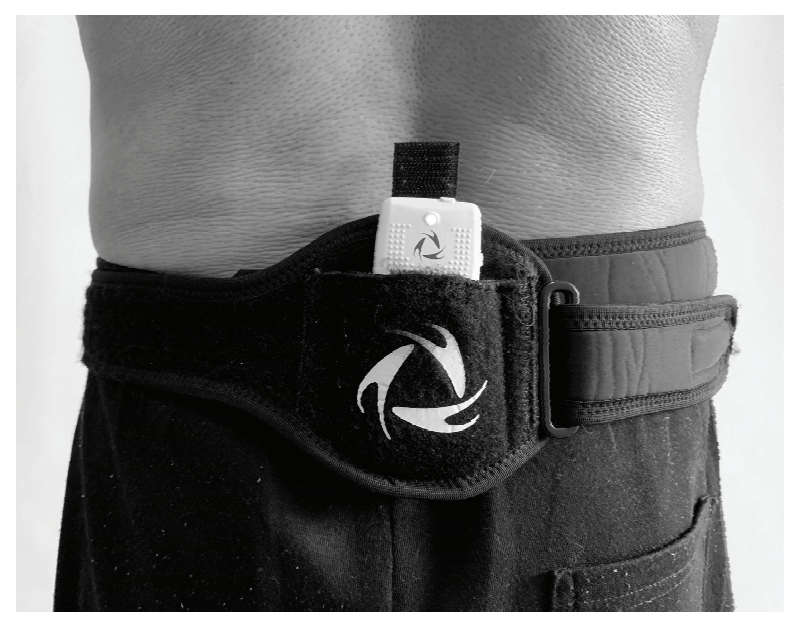

Fig. 1. The assessment of temporo-spatial variables during gait using an inertial sensors

\subsection{Torque analysis}

The professional Biodex System 4 Pro $^{\text {TM }}$ (Biodex Medical Systems Inc., Shirley, NY, USA) was used to assess power-velocity capabilities. People from the study group were asked to sit in the dynamometer chair, which is an integrative part of the Biodex System [19]. The subject's position, with stabilized trunk, pelvis, and thighs, was assured by belts, according to the manufacturer's recommendations, in such way that the knee joint stayed in extension.

A dynamometer construction ensured a precise adjustment of the motion axis (the dynamometer arm) to the tested joint's axis of movement (in this case, the ankle joint). The measurement was conducted at three angular velocities in the sagittal plane and under isometric conditions at the neutral position of the ankle joint. The torque measurement in the static condition (isometry) was also carried out in the ankle joint's neutral position $\left(0^{\circ}=\right.$ perpendicular angle between the shank and the foot), with the foot strapped to the dynamometer platform [20]. Due to differences in the transverse muscle mass of the ankle plantar flexors and dorsiflexors, different angular velocities were assumed for plantar flexion $(30,60$, and $120 \%$ s) and dorsiflexion $(60,120$, and $180 \%$ s) in isokinetic testing.

For each angular velocity, participants were instructed to perform plantarflexor or dorsiflexor maximal voluntary contractions (MVC). Five movement repetitions were done for both isometric and isokinetic measurements. Group testing after a stroke started with an affected limb. For the control group, the first measurement was taken for the lower right and then for the left lower limb. The measurements contained 
one trial at each angular velocity and isometry. The interval between isokinetic measurements was at least one minute between each trial and 30 seconds between isometric repetitions. Before the measurements began, each person was informed, in detail, about his or her course. The patient was also allowed to perform trial tests. Muscle torque was assessed over the entire range of motion in the isokinetic measurements. The torque measurement was first performed for the affected limb. There were no side differences between the torque values of muscles acting on the ankle joints in the control group. The comparative parameters were the relative and absolute peak torques of the healthy limb and the affected limb. One person from the post-stroke group (No. 9) did not agree to the torque measurements.

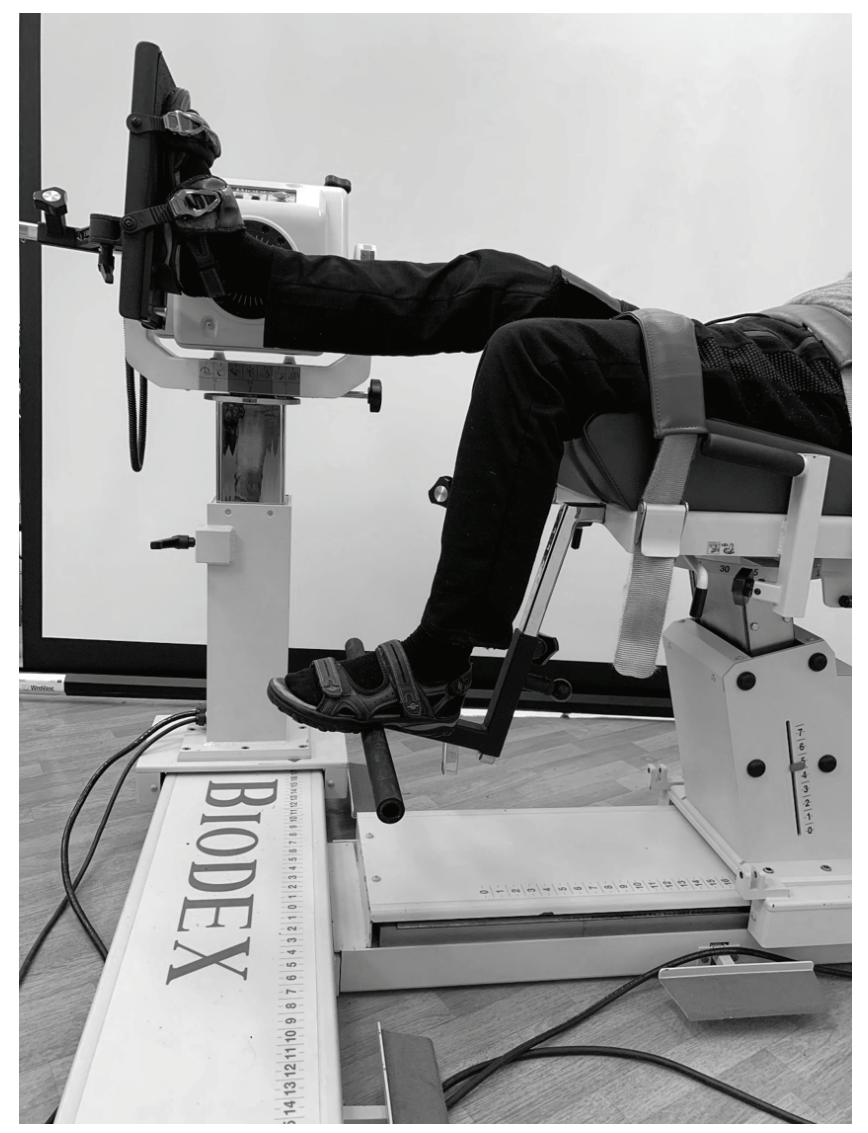

Fig. 2. The assessment of power-velocity capabilities of lower limb using a dynamometer

Muscle torque was analyzed using the Biodex software (Biodex Medical Systems Inc., Shirley, NY, USA). Peak torque was defined as the highest recorded torque value in all conditions assumed for this study. The relative torque was defined as the highest recorded torque value for each of the analyzed conditions in relation to the subject's body weight $[\mathrm{Nm} / \mathrm{kg}]$. The isokinetic torque (dynamic) was evaluated for a given constant angular velocity in the concentric activity. The isometric torque (static) was evaluated as a measurement in a constant angle. The influence of gravity was reduced by using a horizontal position. The G-Studio software, version 3.0.18.0 (BTS Bioengineering Corp., Quincy, MA, USA) was used to assess the temporo-spatial parameters (Fig. 2).

\subsection{Statistical analysis}

Statistical analysis was performed using Statistica software, version 12 (StatSoft, Inc., Tulsa OK, USA). Arithmetic means and standard deviations were calculated for continuous variables. None of the continuous variables had a distribution similar to a normal distribution (based on the Shapiro-Wilk test). Therefore, non-parametric tests were used. The Mann -Whitney $U$-test was used to compare the results of GCAD [steps/min], GSP [m/s], stand-to-sit VTA [m/ $\mathrm{s}^{2}$, and sit-to-stand VTA $\left[\mathrm{m} / \mathrm{s}^{2}\right]$ between the post-stroke and control groups. The Kruskal-Wallis variance analysis and post-hoc tests were used to compare the results of $\mathrm{GC}[\%]$ and torque parameters of the affected and contralateral limbs (post-stroke group) versus the healthy limb (control group). Spearman's test $(R s)$ analyzed the linear association between the results of GSP and torque parameters in both groups. For all analyses, a type 1 error probability of $5 \%(\alpha=0.05)$ was considered, and the obtained $p$-values were rounded to three decimal places for this study.

\section{Results}

\subsection{Gait parameters and the TUG}

The results of the mean temporo-spatial parameters and the TUG in the post-stroke group are presented in Table 3. Post-stroke patients had a statistically significantly lower GCAD compared to healthy participants $(17 \%, p \leq 0.01)$. There was also a difference in single stance phase times during the gait cycle between the affected $(2 \%, p<0.93)$ and contralateral $(7 \%, p<0.99)$ limbs. In post-stroke patients, a gait cycle for the single stance phase was longer for a healthy limb than for an affected limb. For the sit-to-stand task, statistically, significantly lower values of vertical acceleration were found $(42 \%, p<0.03)$ in stroke patients. 
Table 3. Results of time-space parameters for gait and TUG $(\mathrm{M} \pm \mathrm{SD})$

\begin{tabular}{|c|l|c|c|c|}
\hline \multirow{2}{*}{ Test } & \multirow{2}{*}{ Parameter } & \multicolumn{2}{|c|}{ Post-stroke group } & Control group \\
\cline { 3 - 5 } & & Affected limb & Contralateral limb & Healthy limb \\
\hline \multirow{3}{*}{ Gait } & GCAD [steps/min] & \multicolumn{2}{|c|}{$93.3 \pm 17.4^{*}$} & $112.9 \pm 6.1$ \\
\cline { 2 - 5 } & GSP $[\mathrm{m} / \mathrm{s}]$ & \multicolumn{2}{|c|}{$0.9 \pm 0.1$} & $1.09 \pm 0.1$ \\
\cline { 2 - 5 } & GC $[\%]$ & $36.9 \pm 3.2$ & $38.7 \pm 3.7$ & $36.2 \pm 2.3$ \\
\hline \multirow{2}{*}{ TUG } & stand-to-sit VTA $\left[\mathrm{m} / \mathrm{s}^{2}\right]$ & \multicolumn{2}{|c|}{$6.3 \pm 3.5$} & $7.5 \pm 0.7$ \\
\cline { 2 - 5 } & sit-to-stand VTA $\left[\mathrm{m} / \mathrm{s}^{2}\right]$ & \multicolumn{2}{|c|}{$4.1 \pm 1.6^{*}$} & $7.1 \pm 2.3$ \\
\hline
\end{tabular}

Abbreviations: GCAD - gait cadence, GSP - gait speed, GC - a single stance/support time ratio measured as a ratio of a single stance time to gait cycle time and expressed in percent of the affected and contralateral limb, M - mean, SD - standard deviation, TUG - Timed Up and Go test, VTA - average value of vertical acceleration without correction for gravity.

* Statistically significant differences at $p<0.05$.

\subsection{Plantar flexion torque parameters}

The mean values of the torque of ankle plantar flexors, on both the affected and healthy sides of poststroke patients, are presented in Fig. 3. The relative torques of the plantar flexor muscles for the affected and healthy limbs during static conditions were $0.38 \mathrm{Nm} / \mathrm{kg}$ and $0.4 \mathrm{Nm} / \mathrm{kg}$, respectively. They were statistically significantly lower than the torque in the control group, which was $0.96 \mathrm{Nm} / \mathrm{kg}$ (by $63 \%$ and by $58 \%$, $p \leq 0.01)$. The relative torque for the affected and healthy limbs during dynamic conditions at $30 \%$ also yielded a statistically significant difference: $0.36 \mathrm{Nm} / \mathrm{kg}$ for the affected limb, $0.49 \mathrm{Nm} / \mathrm{kg}$ for the healthy limb, and $0.71 \mathrm{Nm} / \mathrm{kg}$ for the control group (by $49 \%$ and by $31 \%, p=0.04)$. In movements at an angular velocity of $60^{\circ} / \mathrm{s}$, the differences were statistically significant between the affected limb, at $0.27 \mathrm{Nm} / \mathrm{kg}$, and the control group, at $0.64 \mathrm{Nm} / \mathrm{kg}$, (by $58 \%, p=0.01$ ). In high-speed movements of $120 \%$ s, statistically significant differences again occurred between the two

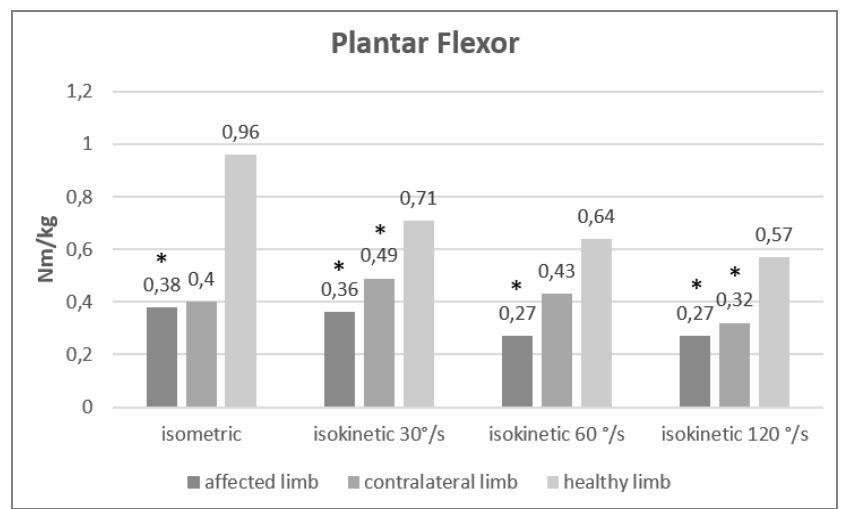

Fig. 3. Plantar flexor torque under static conditions and three angular velocities for the affected and contralateral limbs in post-stroke patients and for the healthy limb. * Statistically significant differences at $p<0.05$ limbs in stroke patients $-0.27 \mathrm{Nm} / \mathrm{kg}$ for the affected side and $0.32 \mathrm{Nm} / \mathrm{kg}$ for the healthy side - and people in the control group $-0.57 \mathrm{Nm} / \mathrm{kg}$ (by $53 \%$ and by $44 \%, p=0.01)$.

\subsection{Dorsiflexor torque parameters}

The dorsiflexor muscles were characterized, as expected, by lower relative torque values compared to plantar flexor muscles (Fig. 4). The only statistically significant difference in the recorded torque was for isometric work of the affected limb, which was $0.3 \mathrm{Nm} / \mathrm{kg}$, compared to the control groups where it was $0.48 \mathrm{Nm} / \mathrm{kg}$ (by $38 \%, p=0.04$ ). Interesting results were observed in the fast dorsiflexion movements of $180^{\circ} / \mathrm{s}$. The affected limb obtained higher relative torque values than the healthy limb (by $15 \%$ and by $14 \%$, $p=0.46, p=0.28)$. In the other tested velocities, no statistically significant differences were recorded, and, as expected, higher relative torque values were recorded for the healthy limb than for the affected limb.

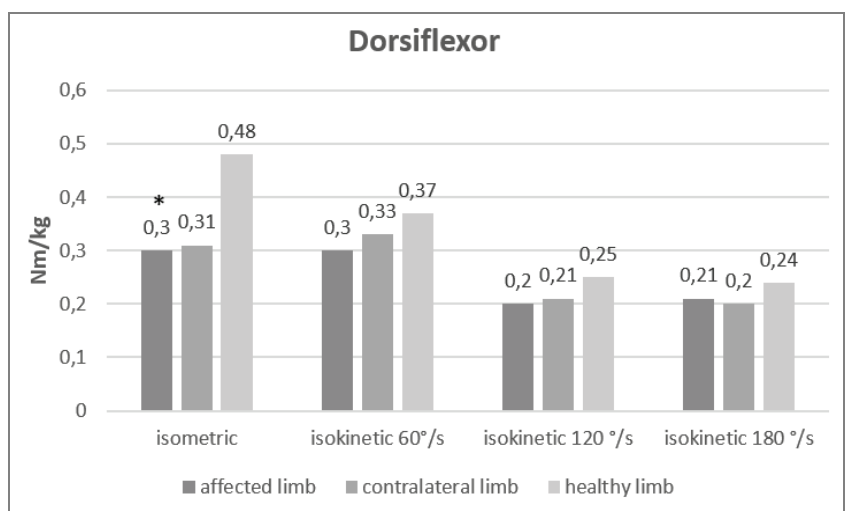

Fig. 4. Dorsiflexion torque under static conditions and three angular velocities for the affected and contralateral limbs in post-stroke patients and for the healthy limb. * Statistically significant differences at $p<0.05$ 


\subsection{Correlations of muscle torque with gait speed}

The next stage of the study analyzed correlations between the torque values of muscles acting on the ankle joint in static and dynamic conditions during plantar flexion and dorsiflexion movements with GSP parameters. A statistically significant positive correlation was observed between the absolute peak torque of the dorsiflexor muscles in static conditions and gait speed $(R s=0.65, p=0.04)$. It was the only statistically significant correlation. Concerning the absolute peak torque of the plantar flexor muscles, the statistical analysis showed a limited positive correlation $(R s=0.53, p=0.11)$. A positive correlation was also demonstrated between GSP and plantar flexor muscles $(R s=0.55, p=0.09)$. However, there were no statistically significant correlations between GSP and dorsiflexor muscle torque $(R s=0.47, p=0.17)$.

\section{Discussion}

Physiotherapy offers a wide range of anti-spastic procedures including neurophysiological methods such as neurodevelopmental treatment (NDT Bobath) or proprioceptive neuromuscular facilitation (PNF), as well as conventional ones constrained-induced movement therapy (CIMT), postural control training, passive stretching, hydrotherapy, hippotherapy, kinesiology taping (KT), and orthopedic devices [21]-[23]. Also, biophysical agents, including neuromuscular stimulation (NMS), electromyography-triggered neuromuscular stimulation (EMG-NMS), transcutaneous electrical nerve stimulation (TENS), functional electrical stimulation (FES), or mechanic therapies such as ultrasound therapy and shock waves (ESWT) are commonly used as supplementary treatments [24]-[27].

Rehabilitation of people after a stroke always starts with a physical examination, especially assessment of gait. In the early stage after stroke, the determination of individual and achievable goals is a priority. In neurological rehabilitation departments, gait assessment is often performed on the basis of visual assessment. In the case of people with low neurological deficits, this assessment may not be sufficient or even flawed. As noted in the introduction, each type of stroke involves to a chronic phase that can last for many years. In this paper, in the study group, despite the low intensity of neurological deficits and the early post-stroke stage, changes in the gait parameters and the torques of muscle acting on the ankle joints were observed.

The aim of the present study was not to assess dependence, but only to assess the power-velocity capabilities of post-stroke patients with early alterations in the motor system. Despite the low intensity of spasticity, differences in the muscle torque production and temporo-spatial parameters were noticeable. At the time of measuring the muscle torque and the selected temporo-spatial parameters, the patients from the post-stroke group were staying in the neurological rehabilitation ward for the first time. The short period after stroke onset - between one and three months - allowed for the assessment of gait and powervelocity capabilities before the formation of compensation phenomena.

A non-standard measurement protocol was used to assess the torque of muscles acting on the ankle joint under isokinetic conditions in the affected and unaffected sides of post-stroke patients. For the high-speed movements of $120 \%$ and $180 \%$ in post-stroke patients, an increase in torque was noted in the affected side. In the control group, the mean relative torque of the ankle plantar flexor and dorsiflexor muscles presented the correct relationship between the torque and angular velocity, i.e., as angular velocity increased, torque decreased. No similar relationship was observed in the group of people after a stroke. In the higher angular velocity values tested, the muscle torque did not decrease with increasing angular velocity. This relationship may correspond to a change in the recruitment of motor units in the muscle groups tested as a consequence of a recent stroke.

In isokinetic testing of the control group, only the values obtained from the plantar flexor muscles differed statistically significantly from those obtained in the post-stroke group. The results published by Kim et al. [6] (ankle plantar flexion of $30 \%$ and $0.19 \mathrm{Nm} / \mathrm{kg}$; dorsiflexion of $30 \%$ and $0.15 \mathrm{Nm} / \mathrm{kg}$ ), by Lomaglio et al. [9] (ankle plantar flexion of $30 \% \mathrm{~s}$ and $0.18 \mathrm{Nm} / \mathrm{kg}$, dorsiflexion of $30 \%$ and $0.11 \mathrm{Nm} / \mathrm{kg}$ ), and by Hsu et al. [28] (ankle plantar flexion of $30 \%$ and $0.21 \mathrm{Nm} / \mathrm{kg}$ ) for the affected side were lower than those obtained in our study(ankle plantar flexion of $30 \%$ and $0.36 \mathrm{Nm} / \mathrm{kg}$ and of $60 \% / \mathrm{s}$ and $0.27 \mathrm{Nm} / \mathrm{kg}$, dorsiflexion of $60 \%$ and $0.25 \mathrm{Nm} / \mathrm{kg}$ and of $120 \%$ and $0.21 \mathrm{Nm} / \mathrm{kg}$ ). Flansbjer et al. [29] noted the largest difference in the affected limb's dorsiflexor muscle torque $-13.5 \mathrm{Nm}$. The papers assessing the relationship of the torque of the muscles acting on the ankle joint to the gait parameters (mainly the GSP) were consistent as to their statistically significant interdependencies [4]. 
In most of the available publications, values for angular velocities of $30 \%$ and $60 \%$ were used for isokinetic testing [6], [22]. The abovementioned angular velocities were selected because they were similar to the peak angular velocity of ankle joints during gait in post-stroke patients. In this study's poststroke group, the gait speed was higher than in previously published studies. Additional differences were the shorter duration from the onset of stroke and the lower degree of AS spasticity on the affected side. Compared to control group, the affected limb in poststroke patients was characterized by a statistically significant difference in torque production of the ankle plantar flexor and dorsiflexor muscles during static conditions.

Using inertial sensors to assess temporo-spatial gait parameters in post-stroke patients enabled quick and reliable assessment of the existing differences. Additionally, the inertial sensors made measurements easy to perform and allowed for valuable analysis of the obtained data. In the present study, people in the post-stroke group had experienced a stroke for the first time, and the study started between one and three months after the onset of stroke. Despite the short period after the neurological incident, disorders characterizing hemiplegia, especially in gait patterns, were recorded. Compared to the reference data from the literature [16], there were statistically significant differences between the GSP, GCAD, and lower limb load symmetry during walking. The patients from the post-stroke group achieved results higher (by 15\% GCAD and by $45 \%$ GSP) than those presented by previous researchers. Milot et al. [32] noted a GSP of 0.62 $\pm 0.26 \mathrm{~m} / \mathrm{s}$ and a GCAD of $81.6 \pm 12.8 \mathrm{steps} / \mathrm{min}$, while Hsu et al. [28] reported a GSP of $0.62 \pm 0.21 \mathrm{~m} / \mathrm{s}$ and a GCAD of $84.5 \pm 14.2 \mathrm{steps} / \mathrm{min}$.

Our results in patients from one to three months post-stroke showed higher GCAD values (Table 3). Also, a single stance/support time ratio measured as a ratio of a single stance time to gait cycle time and expressed in percent of the affected and contralateral limb. This distribution should be approximately $40 \%$ of the swing phase and $60 \%$ of the stance phase [33]. In the study group the proportion of mid stance and swing phases in the gait cycle was changed. The cause of these changes can be detected at a lower gait speed. Additionally, the study group was characterized by a higher gait cadence, which may indicate a compensation of the slower gait speed.

Importantly, in this study's results, there were differences in limb load between the affected and unaffected sides. Patients from the post-stroke group were loaded on the affected side more lightly than on the unaffected side, and these data correspond with results obtained by other authors. Drużbicki et al. [3] noted a $0.96 \pm 1.28 \mathrm{~s}$ stance phase for the affected side and a $1.13 \pm 1.53 \mathrm{~s}$ stance phase for the unaffected side, while Hsu et al. [28] showed a $1.05 \pm 0.31 \mathrm{~s}$ stance phase for the affected side and a $0.94 \pm 0.23 \mathrm{~s}$ stance phase for the unaffected side. It should be also emphasized that the spasticity grade of patients in the present study was between zero and one on the AS. Therefore, concerning hemiplegia severity and time from stroke onset, we noticed differences between this study's obtained values for the measured parameters and the data from previously available research.

The present study has some potential limitations. The research was carried out in the clinical conditions of only one center. The size of the post-stroke group was not large and was not representative enough to generalize changes in ankle muscle torque production to the whole population of patients who have experienced their first stroke with accompanying mild spasticity. Therefore, the pilot character of this study should be emphasized. It should be mentioned that only two patients with hemorrhagic stroke were qualified, however, all participants met the same criteria to be included in the study and they presented similar MAS and BI scores. Therefore, it should be considered that the study group was homogenous at baseline. Another issue to discuss is that the results of this study demonstrated significant asymmetry in the torque production of the plantar flexor and dorsiflexor muscles after a stroke, which may be the reason for negative changes in the selected temporo-spatial parameters of gait. Avoiding high-speed movements for ankle dorsiflexion and implementing strength training should be considered in neurorehabilitation programs.

\section{Conclusions}

The study group consisted of people in the initial period and after the first stroke. Despite the participants of study group having a low level of disability, asymmetries have been noted, which may cause future compensations and their negative consequences. Stroke negatively affects the magnitude of torque asymmetry and changes the selected temporo-spatial parameters for gait and sit-to-stand task. Reduced strength parameters of the muscles acting on the ankle joint are one of the factors that may influence the final gait pattern in post-stroke survivors. Inertial sensors are a valuable method of objectively assessing differences in temporo-spatial parameters in hemiplegic patients. 
Therefore, during everyday neurorehabilitation practice, it is important to introduce methods of strengthening the muscles acting on the ankle joint.

\section{Funding statement}

This study was supported by the Ministry of Science and Higher Education in Poland as a part of a statutory grant of the Wroclaw Medical University in Poland for maintaining research potential (No. ST-962).

\section{Acknowledgements}

This paper has no contributions of specific colleagues, institutions, or agencies that aided the efforts of the authors. The certificated English proofreading services were provided. The Authors wish to thank all of the patients who participated in the study.

\section{References}

[1] Peters D.M., Fridriksson J., Stewart J.C., Richardson J.D., Rorden C., Bonilha L., Middleton A., Gleichgerrcht E., FRITZ S.L., Cortical disconnection of the ipsilesional primary motor cortex is associated with gait speed and upper extremity motor impairment in chronic left hemispheric stroke, Hum. Brain. Mapp., 2018, 39, 120-132.

[2] SÁnchez N., Acosta A.M., LóPEZ-Rosado R., Dewald J.P.A., Neural constraints affect the ability to generate hip abduction torques when combined with hip extension or ankle plantarflexion in chronic hemiparetic stroke, Front. Neurol., 2018, 9, 564.

[3] Drużbicki M., Guzik A., Przysada G., Kwolek A., BrzozowsKa-Magoń A., SobolewsKi M., Changes in gait symmetry after training on a treadmill with biofeedback in chronic stroke patients: a 6-month follow-up from a randomized controlled trial, Med. Sci. Monit., 2016, 22, 4859-4868.

[4] Dias C.P., Freire B., Goulart N.B.A., OnZi E.S., Becker J., Gomes I., ARAmpatzis A., VAZ M.A., Muscle architecture and torque production in stroke survivors: an observational study, Top. Stroke. Rehabil., 2017, 24, 206-213.

[5] SÁnchez N., Acosta A.M., Lopez-Rosado R., Stienen A.H.A., DEWALD J.P.A., Lower extremity motor impairments in ambulatory chronic hemiparetic stroke: evidence for lower extremity weakness and abnormal muscle and joint torque coupling patterns, Neurorehabil. Neural. Repair, 2017, 31, 814-826.

[6] KIM C.M., ENG J.J., The relationship of lower-extremity muscle torque to locomotor performance in people with stroke, Phys. Ther., 2003, 83, 49-57.

[7] Szopa A., Domagalska-Szopa M., LaseK-BAL A., ŻAK A., The link between weight shift asymmetry and gait disturbances in chronic hemiparetic stroke patients, Clin. Interv. Aging, 2017, 12, 2055-2062.

[8] Eng J.J., Lomaglio M.J., Macintyre D.L., Muscle torque preservation and physical activity in individuals with stroke, Med. Sci. Sports. Exerc., 2009, 41, 1353-1360.

[9] LOMAGLIO M.J., ENG J.J., Muscle strength and weight-bearing symmetry relate to sit-to-stand performance in individuals with stroke, Gait Posture, 2005, 22, 126-131.
[10] Moon S.B., Ji Y.-H., Jang H.-Y., Hwang S.-H., Shin D.-B., LeE S.-C., HAN J.-S., HaN C.-S., LeE Y.G., JaNG S.H., PARK S.B., KIM M.J., Gait analysis of hemiplegic patients in ambulatory rehabilitation training using a wearable lower-limb robot: A pilot study, Int. J. Precis. Eng. Manuf., 2017, 18, 1773-1781.

[11] Freire B., Dias C.P., Goulart N.B.A., De Castro C.D., BeCKer J., Gomes I., VAZ M.A., Achilles tendon morphology, plantar flexors torque and passive ankle stiffness in spastic hemiparetic stroke survivors, Clin. Biomech. (Bristol, Avon.), 2017, 41, 72-76.

[12] Rabelo M., Nunes G.S., Da Costa Amante N.M., De Noronha M., FAChin-Martins E., Reliability of muscle strength assessment in chronic post-stroke hemiparesis: a systematic review and meta-analysis, Top. Stroke Rehabil., 2016, $23,26-36$

[13] Fujita K., Hori H., Kobayashi Y., Contribution of muscle activity at different gait phases for improving walking performance in chronic stroke patients with hemiparesis, J. Phys. Ther. Sci., 2018, 30, 1381-1385.

[14] Titus A.W., Hillier S., Louw Q.A., Inglis-Jassiem G., $A n$ analysis of trunk kinematics and gait parameters in people with stroke, Afr. J. Disabil, 2018, 7, 310.

[15] Von Elm E., Altman D.G., Egger M., Pocock S.J., GøTZSCHE P.C., VANDENBROUCKE J.P., STROBE INITIATIVE, Strengthening the Reporting of Observational Studies in Epidemiology (STROBE) statement: guidelines for reporting observational studies, BMJ, 2007, 335, 806-808.

[16] Harbo T., Brincks J., ANDERSEN H., Maximal isokinetic and isometric muscle strength of major muscle groups related to age, body mass, height, and sex in 178 healthy subjects, Eur. J. Appl. Physiol., 2012, 112, 267-275.

[17] SANKARPANDi S.K., BALDWIn A.J., RAY J., MAzzÀ C., Reliability of inertial sensors in the assessment of patients with vestibular disorders: a feasibility study, BMC Ear. Nose. Throat. Disord., 2017, 17, 1.

[18] Schenkman M., Berger R.A., Riley P.O., Mann R.W., HODGE W.A., Whole-body movements during rising to standing from sitting. Phys. Ther., 1990, 70, 638-648.

[19] TankeVicius G., Lankaite D., Krisciunas A., Test-retest reliability of biodex system 4 pro for isometric ankle-eversion and -inversion measurement, J. Sport Rehabil., 2013, 22, 212-215.

[20] Gonçalves G.H., Sendín F.A., Da Silva Serrão P.R.M., Selistre L.F.A., Petrella M., Carvalho C., Mattiello S.M., Ankle strength impairments associated with knee osteoarthritis, Clin. Biomech. (Bristol, Avon.), 2017, 46, 33-39.

[21] Dymarek R., Ptaszkowski K., SŁupska L., Paprocka-Borowicz M., Taradaj J., Halski T., RosińczuK J., Poststroke spasticity management including a chosen physiotherapeutic methods and improvements in motor control - review of the current scientific evidence, Wiad. Lek., 2017, $70,357-365$.

[22] Monaghan K., Horgan F., Blake C., Cornall C., Hickey P.P., Lyons B.E., LANGHORNe P., Physical treatment interventions for managing spasticity after stroke, Cochrane. Database. Syst. Rev., 2017, 2, 23.

[23] Hatem S.M., Saussez G., Della Faille M., Prist V., Zhang X., DisPa D., BleYeNheufT T., Rehabilitation of motor function after stroke: a multiple systematic review focused on techniques to stimulate upper extremity recovery, Front. Hum. Neurosci., 2016, 10, 24.

[24] Slupska L., Halski T., Żytkiewicz M., Ptaszkowski K., Dymarek R., Taradaj J., Paprocka-Borowicz M., Proprio- 
ceptive neuromuscular facilitation for accessory respiratory muscles training in patients after ischemic stroke, Adv. Exp. Med. Biol., 2019, 1160, 81-91.

[25] Dymarek R., Ptaszkowski K., SŁupska L., Halski T., TARADAJ J., RosińCZUK J., Effects of extracorporeal shock wave on upper and lower limb spasticity in post-stroke patients: A narrative review, Top. Stroke. Rehabil., 2016, 26, $1-11$.

[26] Dymarek R., TARAdaj J., Rosińczuk J., Extracorporeal shockwave stimulation as alternative treatment modality for wrist and fingers spasticity in poststroke patients: a prospective, open-label, preliminary clinical trial, Evid. Based. Complement. Alternat. Med., 2016, 27.

[27] Dymarek R., TARAdaj J., RosińczuK J., The Effect of Radial Extracorporeal Shockwave stimulation on upper limb spasticity in chronic stroke patients: a single-blind, randomized, placebo-controlled study, Ultrasound. Med. Biol., 2016, 42, $1862-1875$.

[28] Hsu A.-L., TAng P.-F., JAN M.-H., Analysis of impairments influencing gait velocity and asymmetry of hemiplegic pa- tients after mild to moderate stroke, Arch. Phys. Med. Rehabil., 2003, 84, 1185-1193.

[29] Flansbjer U.-B., Miller M., Downham D., LeXell J., Progressive resistance training after stroke: effects on muscle strength, muscle tone, gait performance and perceived participation, J. Rehabil. Med., 2008, 40, 42-48.

[30] Gao F., Grant T.H., Roth E.J., Zhang L.-Q., Changes in passive mechanical properties of the gastrocnemius muscle at the muscle fascicle and joint levels in stroke survivors, Arch. Phys. Med. Rehabil., 2009, 90, 819-826.

[31] CRUZ T.H., DHAHER Y.Y., Evidence of abnormal lower-limb torque coupling after stroke: an isometric study, Stroke, 2008, 39, 139-147.

[32] Milot M.-H., Nadeau S., Gravel D., Bourbonnais D., Effect of increases in plantarflexor and hip flexor muscle strength on the levels of effort during gait in individuals with hemiparesis, Clin. Biomech. (Bristol, Avon.), 2008, 23, 415-423.

[33] Pietraszewski B., Winiarski S., Jaroszczuk S., Three-dimensional human gait pattern - reference data for normal men, Acta. Bioeng. Biomech., 2012, 14, 9-16. 